\title{
COVID-19 e ambiente alimentar digital no Brasil: reflexões sobre a influência da pandemia no uso de aplicativos de delivery de comida
}

\author{
COVID-19 and the digital food environment in \\ Brazil: reflections on the pandemic's influence \\ on the use of food delivery apps
}

\section{COVID-19 y ambiente alimentario digital en Brasil: reflexiones sobre la influencia de la pandemia en el uso de aplicaciones de entrega de comida}

Laís Vargas Botelho 1

Letícia de Oliveira Cardoso 1

Daniela Silva Canella 2

doi: 10.1590/0102-311X00148020
A COVID-19 é uma doença infecciosa causada pelo recém-descoberto SARS-CoV-2, para a qual ainda não há vacina ou tratamento comprovadamente eficaz disponível 1. Considerando que esse coronavírus tem alta taxa de transmissibilidade e que sua transmissão se dá por contato pessoal próximo ou com superfícies contaminadas 2, o distanciamento físico é a melhor estratégia disponível para enfrentar sua disseminação. Por isso, desde que foi declarada a transmissão comunitária da COVID-19 no Brasil, atividades comerciais não essenciais foram interrompidas, e as essenciais tiveram de adotar regras específicas de funcionamento ${ }^{3}$.

Até o momento da redação deste artigo não havia indicativo de redução do número de casos e óbitos diários no nível nacional. Apesar disso, iniciativas governamentais de flexibilização do distanciamento físico avançavam em diversos estados e municípios, diminuindo as restrições ao comércio outrora adotadas 1 .

$\mathrm{O}$ atendimento presencial em estabelecimentos do setor de alimentação fora do lar foi proibido ou limitado por decretos durante a maior parte do tempo desde o início da pandemia 4,5. Como consequência, bares, lanchonetes, restaurantes e congêneres adotaram ou intensificaram sua presença no ambiente digital, principalmente em aplicativos de delivery de comida (neste texto, adotaremos simplificadamente este termo para designar o serviço de entrega de alimentos e bebidas prontos para consumo, ou seja, que dispensam qualquer etapa de preparo pelo consumidor) 6 . Por sua vez, clientes interessados em comida preparada fora do lar também passaram a recorrer mais aos serviços de delivery e take away (para retirada no local) nesses aplicativos 7,8.

Pelos motivos mencionados, é possível que o distanciamento físico durante a pandemia de COVID-19 esteja contribuindo para catalisar a ampliação do uso de aplicativos de delivery de comida no Brasil. Por isso, a partir deste momento de crise sanitária, cabe refletir sobre possíveis repercussões da difusão da prática de comprar comida online em aplicativos para a saúde e a nutrição.

\section{O delivery de comida e a saúde do consumidor}

Aplicativos de delivery integram o ambiente alimentar digital ${ }^{9}$, pois são canais de conveniência para a realização de compras online 10 . Nesse ambiente, as possibilidades de local de compra são ampliadas, o que melhora a disponibilidade e a acessibilidade aos alimentos preparados fora do lar 11. Contu-
1 Escola Nacional de Saúde Pública Sergio Arouca, Fundação Oswaldo Cruz, Rio de Janeiro, Brasil. 2 Centro Biomédico, Universidade do Estado do Rio de Janeiro, Rio de Janeiro, Brasil.

Correspondência L. V. Botelho Rua Domingos do Carmo Medeiros 180, Macaé, RJ 27973-543, Brasil. lvbotelho12@gmail.com 
do, a saúde do consumidor pode ser afetada negativamente visto que a composição nutricional dos alimentos e bebidas disponíveis em tais plataformas deve ser semelhante à daqueles presentes nos estabelecimentos físicos 12. Como a alimentação fora do lar faz parte da dieta do brasileiro e apresenta perfil nutricional alto em energia e pobre em nutrientes 13 , a aquisição de comida em aplicativos pode contribuir para o consumo de alimentos não saudáveis e para o desenvolvimento de doenças crônicas não transmissíveis associadas 12 .

Segundo pesquisa realizada em Belo Horizonte (Minas Gerais), cerca de 80\% dos estabelecimentos presentes nos dois aplicativos mais utilizados vendiam bebidas ultraprocessadas, $38 \%$ vendiam lanches ultraprocessados e 33\%, produtos de sorveteria. Em contrapartida, menos de 16\% comercializavam refeições com predominância de legumes e verduras, e somente $4 \%$ vendiam frutas ou preparações baseadas em frutas (observações não publicadas) ${ }^{14}$. Sabe-se também que preparações culinárias baseadas em alimentos ultraprocessados, como pizzas, hambúrgueres e outros tipos de lanches, figuram entre as categorias de comida mais pedidas nas plataformas 15,16. Portanto, o ambiente digital dos aplicativos de delivery de comida pode ser entendido como um pântano alimentar ${ }^{17}$. Afinal, como não depende da distância física entre os estabelecimentos e os clientes, concentra maior proporção de pontos de venda de alimentos não saudáveis em comparação com alimentos saudáveis.

Outro fator que deve ser considerado é que a dinâmica das escolhas alimentares realizadas online difere da experiência de compra em estabelecimentos físicos. Por exemplo, estudos sobre compra de itens de supermercado pela Internet sugerem queda na aquisição de alimentos não saudáveis por impulso, mas também há evidências de hesitação na compra de alimentos frescos 18 . Apesar da escassez de estudos semelhantes que analisem a aquisição de comida pronta para consumo online, aplicativos de comida têm particularidades que podem direcionar o consumidor para alimentos não saudáveis. Sua interface simula uma praça de alimentação em que determinadas opções podem ser mais atrativas devido a imagens ilustrativas; promoções; combos; entrega gratuita; e, em especial, devido à programação algorítmica, que evidencia alimentos e ofertas com base nas compras pregressas e no que pessoas com perfil semelhante têm maior predisposição a pedir.

\section{Intensificação do uso de aplicativos de delivery de comida durante a pandemia}

A expansão do acesso à Internet e aos dispositivos móveis inteligentes e a disseminação da cultura digital são fatores apontados como contribuintes para a popularização do uso de aplicativos de delivery de comida no Brasil 10. Os 26,6 milhões de pedidos entregues em novembro de 2019 pela iFood, empresa líder do setor na América Latina, ilustram essa expansão 19.

Segundo Bezerra et al. 13, a frequência de consumo de alimentos fora do lar no Brasil tem relação positiva com a renda e negativa com a idade. Semelhantemente, a proporção de usuários de aplicativos de delivery de comida é maior entre adultos jovens, nas classes socioeconômicas A e B (60\%) e na região com a maior concentração de renda, o Sudeste (61\%) 20. Além disso, nas capitais e regiões metropolitanas o delivery online compreende uma proporção consideravelmente maior $(39,5 \%)$ do mercado de entrega em comparação com o interior dos estados (6,9\%) 16.

O presente momento de distanciamento físico pode estar catalisando a adoção do delivery de comida nas cidades onde esta opção está disponível porque, desde que os serviços de alimentação sigam as orientações higienicossanitárias 21, usar esta solução tecnológica é mais seguro do que sair para comer. Assim, mais brasileiros estão optando por receber comida em domicílio. Para mais, os aplicativos têm investido em estratégias como descontos, promoções e frete grátis durante esse período 6.

As empresas de delivery não têm divulgado dados consolidados sobre o impacto da pandemia em suas vendas. Contudo, a iFood, por exemplo, confirmou que houve um aumento na demanda por delivery acompanhando o espalhamento da COVID-19. Isso é, começou em São Paulo e no Rio de Janeiro, alcançou outras capitais e depois se interiorizou. Adicionalmente, seus clientes passaram a comprar maiores quantidades por pedido, com maior frequência e em momentos diferentes daqueles em que geralmente ocorriam picos de consumo. Isso inclui os cafés da manhã e da tarde e o almoço - neste caso, uma importante explicação apontada é a possibilidade de pagamento com o cartão refeição 8. 
Apesar de os usuários de aplicativos de delivery de comida apresentarem um perfil socioeconômico privilegiado em comparação com a totalidade da população brasileira, as questões aqui colocadas não se restringem às pessoas com maior poder aquisitivo. Mesmo antes da pandemia, empresas de delivery de comida investiam em ações para diversificar seu público, como o programa que digitalizou o "prato feito" em 40 cidades brasileiras, com entrega de marmitas a partir de R\$10,00. Além do aumento de vendas, essa iniciativa visou a atrair mais usuários das classes C e D 22.

Ademais, a indústria de delivery de comida adota estratégias similares àquelas que a indústria alimentícia tem usado para expandir o mercado de alimentos ultraprocessados em países de média e baixa renda 23 . Dentre essas, se destacam a captação de investimentos milionários; a aquisição de contrapartes menores em outros países; e o alto investimento em publicidade 24 . Sendo assim, a exemplo da intensificação da aquisição de alimentos ultraprocessados no estrato da população com menor renda 25 , é possível que classes socioeconômicas mais baixas também passem a comprar comida no ambiente digital com maior frequência.

Relativamente ao momento atual, acredita-se que, no processo de atração e fidelização de clientes que não usavam plataformas de delivery regularmente, estejam sendo alcançados públicos com perfis diferentes dos habituais. Inclusive pessoas mais velhas, que são grupo de risco para a COVID-19, podem estar experimentando comprar comida online 7 .

\section{Considerações finais}

No âmbito da nutrição em saúde pública, medidas têm sido defendidas para desestimular o consumo de alimentos ultraprocessados, como a adoção de embalagens com advertência frontal, a tributação de bebidas açucaradas e a regulação da publicidade e de ambientes 26 . Contudo, a despeito da rápida ascensão da indústria de delivery de comida, que pode estar sendo impulsionada no contexto da pandemia, ainda não existem propostas de medidas específicas para estimular a aquisição de preparações culinárias baseadas em alimentos in natura ou minimamente processados, e desencorajar a compra daquelas à base de alimentos ultraprocessados.

Nesse sentido, a adoção de algumas intervenções poderia proteger a saúde. Por exemplo, proibir a entrega de comida em escolas; sobretaxar combos vendidos a preços inferiores à soma dos valores de seus componentes, e porções grandes cujos preços representam vantagem financeira sobre a compra de porções menores; regulamentar o uso de algoritmos de recomendação; e regular o uso do cartão refeição no âmbito do Programa de Alimentação do Trabalhador.

A indústria de delivery de comida provavelmente sairá fortalecida da crise sanitária, e adquirir comida online, especialmente via aplicativos, poderá integrar o cotidiano de muitos brasileiros após a pandemia. Dessa maneira, embora isso esteja fora do escopo deste artigo, salienta-se a necessidade de atenção e aprofundamento em diversos aspectos da saúde pública intrincados a esse fenômeno, como os direitos trabalhistas dos entregadores e o impacto ambiental decorrente do uso de embalagens para transportar refeições. No que se refere à saúde do consumidor, advoga-se pela necessidade de estudos sobre a prática de comprar comida em aplicativos a fim de identificar potenciais impactos nutricionais, definir e fundamentar ações prioritárias para promover uma alimentação adequada e saudável nesse canal digital. 


\section{Colaboradores}

L. V. Botelho contribuiu com a concepção, redação, revisão crítica e aprovação da versão final do manuscrito. L. O. Cardoso e D. S. Canella contribuíram com a redação, revisão crítica e aprovação da versão final do manuscrito.

\section{Informações adicionais}

ORCID: Laís Vargas Botelho (0000-0003-28101922); Letícia de Oliveira Cardoso (0000-00031312-1808); Daniela Silva Canella (0000-00019672-4983).

\section{Referências}

1. Rafaell RMR, Neto M, Carvalho MMB, David HMSL, Acioli S, Faria MGA. Epidemiologia, políticas públicas e pandemia de Covid-19: o que esperar no Brasil? Rev Enferm UERJ 2020; 28:e49570.

2. World Health Organization. Q\&A on coronaviruses (COVID-19). https://www.who.int/newsroom/q-a-detail/q-a-coronaviruses (acessado em 23/Mai/2020).

3. Brasil. Decreto no 10.282 , de 20 de março de 2020. Regulamenta a Lei no 13.979, de 6 de fevereiro de 2020, para definir os serviços públicos e as atividades essenciais. Diário Oficial da União 2020; 20 mar.

4. Decretos do Governo de SP com medidas de prevenção e combate ao novo coronavírus. SP Notícias 2020; 26 mar. https://www.saopaulo. sp.gov.br/spnoticias/decretos-do-governode-sp-com-medidas-de-prevencao-e-combate -ao-novo-coronavirus/.

5. Rio de Janeiro. Decreto no 47.102, de 1o de junho de 2020. Dispõe sobre as medidas de enfrentamento da propagação do novo coronavírus (COVID-19), em decorrência da situação de emergência em saúde, e dá outras providências. Diário Oficial do Estado do Rio de Janeiro 2020; 2 jun.

6. Soupin E. Com cariocas de quarentena, pedidos de delivery disparam no Rio. G1 2020; 19 mar. https://g1.globo.com/rj/rio-de-janeiro/noti cia/2020/03/19/com-cariocas-de-quarentenapedidos-de-delivery-disparam-no-rio.ghtml.

\section{Agradecimentos}

L. V. Botelho é bolsista do Programa de Excelência Acadêmica (CAPES/MEC).
7. 99 Food. Serviço ganha novos públicos e ajuda a manter bares e restaurantes em operação durante a pandemia. Gazeta do Povo 2020; 29 abr. https://www.gazetadopovo.com.br/con teudo-publicitario/99-food/servico-ganha-no vos-publicos-e-ajuda-a-manter-bares-e-res taurantes-em-operacao-durante-a-pandemia/.

8. Frabasile D, Campos E, Buldrini R. Coronavírus: como o iFood está lidando com o aumento da demanda por delivery. Época Negócios 2020; 3 abr. https://epocanegocios.globo.com/ Startup/noticia/2020/04/coronavirus-comoo-ifood-esta-lidando-com-o-aumento-da-de manda-por-delivery.html.

9. Granheim SI. The digital food environment. UNSCN Nutrition 2019; 44:116-21.

10. Pigatto G, Machado JGCF, Negreti AS, Machado LM. Have you chosen your request? Analysis of online food delivery companies in Brazil. $\mathrm{Br}$ Food J 2017; 119:639-57.

11. Maimaiti M, Zhao X, Jia M, Ru Y, Zhu S. How we eat determines what we become: opportunities and challenges brought by food delivery industry in a changing world in China. Eur J Clin Nutr 2018; 72:1282-6.

12. Keeble M, Adams J, Sacks G, Vanderlee L, White CM, Hammond D, et al. Use of online food delivery services to order food prepared awayfrom-home and associated sociodemographic characteristics: a cross-sectional, multi-country analysis. Int J Environ Res Public Health 2020; 17:5190. 
13. Bezerra IN, Souza AM, Pereira RA, Sichieri R. Consumo de alimentos fora do domicílio no Brasil. Rev Saúde Pública 2013; 47 Suppl 1:200s-11.

14. Horta PM, Souza JPM, Rocha LL, Mendes LL. Digital food environment of a Brazilian metropolis: food availability and marketing strategies used by delivery apps. Public Health Nutr 2020; (Online ahead of print).

15. iFood; Box 1824. A revolução da alimentação na era digital. https://revolucaodelivery.ifood. com.br/\#/ (acessado em 14/Mai/2019).

16. Delivery Much. Diagnóstico do delivery de comida no interior do Brasil em 2018. https:// blog.deliverymuch.com.br/delivery-muchfranquia/ (acessado em 24/Mai/2020).

17. Mui Y, Jones-Smith J, Thornton R, Porter KP, Gittelsohn J. Relationships between vacant homes and food swamps: a longitudinal study of an urban food environment. Int J Environ Res Public Health 2017; 14:1426.

18. Pitts SBJ, Ng SW, Blitstein JL, Gustafson A, Niculescu M. Online grocery shopping: promise and pitfalls for healthier food and beverage purchases. Public Health Nutr 2018; 21:336076.

19. iFood chega a 26,6 milhões de pedidos no mês de novembro. E-Commerce Brasil 2019; 16 dez. https://www.ecommercebrasil.com.br/ noticias/ifood-chega-a-266-milhoes-de-pedi dos-no-mes-de-novembro/ (acessado em 30/ $\mathrm{Jul} / 2020)$.

20. Instituto Qualibest. Consumo de comida delivery por aplicativo. https://www.instituto qualibest.com/download/uso-de-apps-de-deli very-de-comida/ (acessado em 24/Mai/2020).
21. Associação Brasileira de Bares e Restaurantes. Cuidados de higiene no delivery para bares e restaurantes na crise do novo coronavírus. https://drive.google.com/file/d/1u3vSZDLA qDJeVTLROp5WM5WcTED 1 tpKV/view (acessado em 24/Mai/2020).

22. Wolf G. iFood Loop: como a startup de refeições quer 'digitalizar o PF'. O Estado de S. Paulo 2020; 29 jan. https://link.estadao.com.br/noti cias/inovacao,ifood-loop-como-a-startup-de -refeicoes-quer-digitalizar-o-pf,70003176441.

23. Monteiro CA, Cannon G. The impact of transnational "Big Food" companies on the South: a view from Brazil. PLoS Med 2012; 9:e1001252.

24. Bertão N. Com apetite de unicórnio, iFood quer se reinventar. Exame 2017; 14 dez. https:// exame.com/revista-exame/com-apetite-deunicornio/.

25. Instituto Brasileiro de Geografia e Estatística. POF 2017-2018: alimentos frescos e preparações culinárias predominam no padrão alimentar nacional. Agência IBGE Notícias 2020; 3 abr. https://agenciadenoticias.ibge.gov.br/ agencia-sala-de-imprensa/2013-agencia-denoticias/releases/27298-pof-2017-2018-ali mentos-frescos-e-preparacoes-culinarias-pre dominam-no-padrao-alimentar-nacional.

26. Swinburn BA, Kraak VI, Allender S, Atkins VJ, Baker PI, Bogard JR, et al. The global syndemic of obesity, undernutrition, and climate change: the Lancet Commission report. Lancet 2019; 393:791-846.
Recebido em 09/Jun/2020

Versão final reapresentada em 11/Ago/2020

Aprovado em 17/Ago/2020 